\title{
Fuzzy and SVM based Power System Stabilizer for Single Machine Infinite Bus System
}

\author{
Bhavans Shah \\ Assistant Professor \\ Sardar Patel College of Engineering, \\ V. V. Nagar, Gujarat
}

\author{
Prashant Chaudhari \\ Lecturer \\ $B$ \& B Institute of Technology, \\ V. V. Nagar, Gujarat
}

\begin{abstract}
Power system stabilizers (PSSs) are used to enhance the damping during low frequency oscillations. Artificial intelligence techniques provide one alternative for stability enhancement and speed deviation $(\Delta \mathrm{w})$. In this paper we have applied Fuzzy based and Support Vector Machine (SVM) based approach to PSS for Single Machine Infinite Bus (SMIB) System .The proposed method using SVM techniques achieves better improvement than Fuzzy Based power system stabilizer with reference to Conventional PSS with same condition applied. In the present paper, Fuzzy based PSS Simulink model using triangular membership function (FPSS) and novel approach for on-line adaptive tuning of Support Vector Machine based Power System Stabilizer (SVMPSS) using sigmoid kernel function is presented. The simulation results of the proposed SVMPSS and FPSS are compared to those of conventional stabilizers in for a SMIB system. The results show the Robustness of the proposed SVMPSS and its ability to enhance system damping over a wide range of operating conditions and system parameter variations.
\end{abstract}

\section{Keywords}

Power system stabilizer, SMIB System, Fuzzy logic, Support Vector Machine, Dynamic stability, SIMULINK

\section{INTRODUCTION}

Power system stability is a property of a power system that enables it to remain in a state of operating equilibrium under normal operating conditions. Small signal and transient are two categories of stability. Small signal stability is the ability of the system to return to a normal operating state following a small disturbance. Transient stability is the ability of the system to return a normal operating state following a severe disturbance, such as a single or multi-phase short-circuit or a generator loss.

Low frequency oscillations are a major problem in large power system. A power system stabilizer provides supplementary control signal to the excitation system of electric generating unit for damping these low frequency oscillation. Power system stabilizers are successfully used in power systems for few years because of their flexibility low cost and easy implementation.

The power system stabilizer is used to generate supplementary control signal in order to dampen the low frequency oscillation. The conventional power system stabilizer is widely used in existing power system and has contributed to the enhancement of the dynamic stability of power systems.

The parameters of conventional power system stabilizer are based on linearized model of power system around of nominal operating point. Power systems are highly nonlinear systems so the design of conventional Power system stabilizer based on liberalized model of the power systems cannot guarantee its performance in practical operating environment. To improve the performance of conventional power system stabilizer many techniques have been proposed for the design for example genetic algorithm, neural network, fuzzy logic, SVM and many other intelligent optimization techniques.

The paper presents the performance of single machine infinite bus system with Fuzzy Logic based power system stabilizer with triangular membership function is used. Here we have taken speed deviation $(\Delta w)$ and acceleration as input variables to fuzzy logic controller and voltage parameter considered as output variable then SVM bases approach with kernel function is considered. The simulations are implemented in SIMULINK environment.

\section{SYSTEM DESIGN}

\subsection{Synchronous Machine Model:}

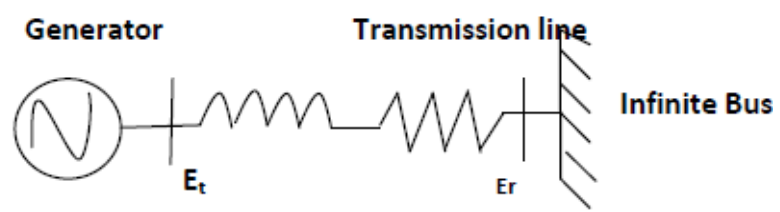

Fig 1: Synchronous Machine Connected to Infinite Bus

\subsection{Excitation System:}

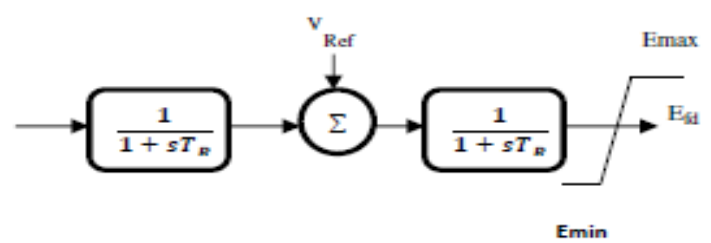

Fig 2 :Block Diagram of Excitation System

Excitation system is capable of responding rapidly to a disturbance so as to enhance transient stability and of modulating the generator field so as to enhance small scale stability

\subsection{Power System Stabilizer (PSS):}

Power system stabilizers (PSS) were developed to aid in damping these oscillations via modulations of excitation system of generator s. The action of a PSS is to extend the angular stability limits of a power system by providing supplemental damping to the oscillation of synchronous machine rotors through the generator excitations. 


\section{FUZZY LOGIC BASED PSS}

The variables chosen for this controller are speed deviation, acceleration and voltage. In this, the speed deviation and acceleration are the input variables and voltage is the output variable. The number of linguistic variables describing the fuzzy subsets of a variable varies according to the application. Usually an odd number is used. A reasonable number is seven. However, increasing the number of fuzzy subsets results in a corresponding increase in the number of rules. Each linguistic variable has its fuzzy membership function. The membership function maps the crisp values into fuzzy variables. The triangular membership functions are used to define the degree of membership. It is important to note that the degree of membership plays an important role in designing a fuzzy controller.

Each of the input and output fuzzy variables are assigned seven linguistic fuzzy subsets varying from negative big (NB) to positive big (PB). Each subset is associated with a triangular membership function to form a set of seven membership functions for each fuzzy variable.

Table 1. Membership functions for fuzzy variables

\begin{tabular}{|l|l|}
\hline NB & NEGATIVE BIG \\
\hline NM & NEGATIVE MEDIUM \\
\hline NS & NEGATIVE SMALL \\
\hline ZE & ZERO \\
\hline PS & POSITIVE SMALL \\
\hline PM & POSITIVE MEDIUM \\
\hline PB & POSITIVE BIG \\
\hline
\end{tabular}

\subsection{Fuzzy Rule Base}

A set of rules which define the relation between the input and output of fuzzy controller can be found using the available knowledge in the area of designing PSS. These rules are defined using the linguistic variables. The two inputs, speed and acceleration, result in 49 rules for each machine. The typical rules are having the following structure:

1) Rule 1: If speed deviation is NM (negative medium) AND acceleration is PS (positive small) then voltage (output of fuzzy PSS) is NS (negative small).

2) Rule 2: If speed deviation is NB (negative big) AND acceleration is NB (negative big) then voltage output of fuzzy PSS) is NB (negative big).

3) Rule 3: If speed deviation is PS (positive small) AND acceleration is PS (positive small) then voltage (output of fuzzy PSS) is PS (positive small). And so on.

Table 2. Fuzzy Rule

\begin{tabular}{|l|l|l|l|l|l|l|l|}
\hline \multirow{2}{*}{$\begin{array}{l}\text { Speed } \\
\text { Deviation }\end{array}$} & \multicolumn{6}{|l|}{ Acceleration } \\
\cline { 2 - 8 } & NB & NM & NS & ZE & PS & PM & PB \\
\hline NB & NB & NB & NB & NB & NM & NM & NS \\
\hline NM & NB & NM & NM & NM & NS & NS & ZE \\
\hline NS & NM & NM & NS & NS & ZE & ZE & PS \\
\hline ZE & NM & PS & NS & ZE & PS & PS & PM \\
\hline PS & NS & ZE & ZE & PS & PS & PM & PM \\
\hline PM & ZE & PS & PS & PM & PM & PM & PB \\
\hline PB & PS & PM & PM & PB & PB & PB & PB \\
\hline
\end{tabular}

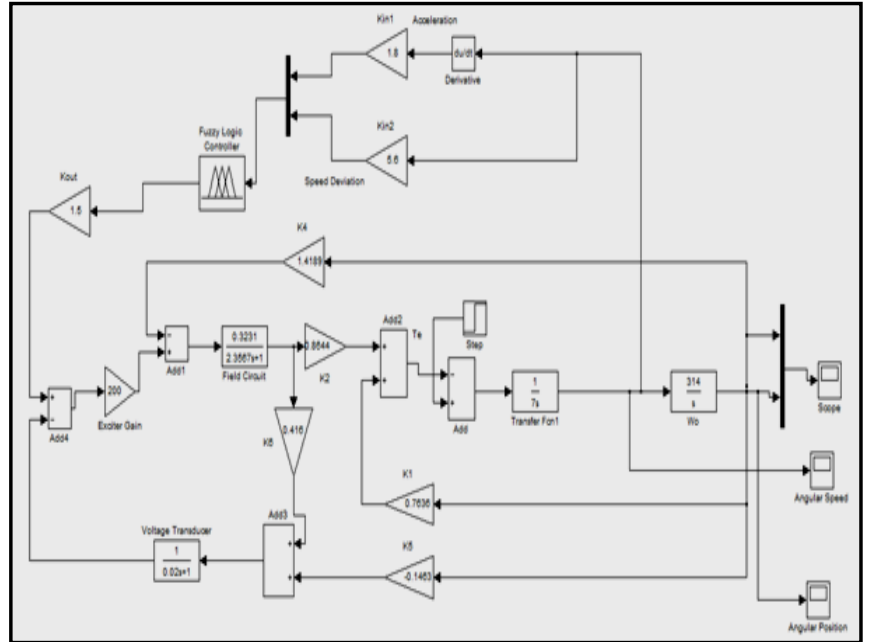

Fig 3: Block Diagram of Fuzzy Logic Controller Implemented on Single Machine Infinite Bus

The figure 3 shows the diagram of the representation of fuzzy based controller for single machine infinite bus system. Here the angular velocity and its derivative are inputs and voltage is output. Kin1, Kin2 and Kout gains which normalize inputs and output according to the range in which the membership functions are defined. The gain parameters are tuned to give the desired response.

\section{SVM BASED PSS}

\subsection{Kernel Function in Support Vector Machine}

The idea of the kernel function is to enable operations to be performed in the input space rather than the potentially high dimensional feature space. Hence the inner product does not need to be evaluated in the feature space. We want the function to perform mapping of the attributes of the input space to the feature space. The kernel function plays a important role in SVM and its performance. It is based upon reproducing Kernel Hilbert Spaces

$\mathrm{K}\left(\mathrm{x}, \mathrm{x}^{\prime}\right)=\left\{\varphi(\mathrm{x}), \varphi\left(\mathrm{x}^{\prime}\right)\right\}$

If $\mathrm{K}$ is a symmetric positive definite function, which satisfies Mercer's Conditions

$\mathrm{K}\left(\mathrm{x}, \mathrm{x}^{\prime}\right)=\sum_{m}^{\infty} \alpha \mathrm{m} \phi \mathrm{m}(\mathrm{x}) \phi \mathrm{m}\left(\mathrm{x}^{\prime}\right) \quad \alpha \mathrm{m} \geq 0$

$\iint K\left(\mathrm{x}, \mathrm{x}^{\prime}\right) \mathrm{g}\left(\mathrm{x}^{\prime}\right) \mathrm{dx} \mathrm{dx} \mathrm{x}^{\prime}>0$

Then the kernel represents a legitimate inner product in feature space. The training set is not linearly separable in an input space but it is linearly separable in the feature space.

\subsection{Choosing Kernel and Corresponding Parameters}

In the SVR algorithm, the kernel function, its parameters, and SVR training parameters $(\mathrm{C}$, and $\varepsilon$ ) for $\varepsilon$-insensitive loss function play a key role in the SVR performance. Parameter C is the tradeoff between model complexity (flatness) and the degree of deviations allowed in the optimization formulation. Parameter $\varepsilon$ controls the width of the $\varepsilon$-insensitive zone, which affects the number of support vectors used to construct the regression function. The kernel function represents the mapping instrument that is necessary to transform the nonlinear input space to a high-dimensional feature space where linear regression is possible. The mapping depends on the 
intrinsic topological structure of the data and applicationdomain knowledge. It implies that the kernel type and all parameters need be optimally chosen to get the best performance. However, there are no structural methods for determining efficiently the selection of kernel and all parameters. Moreover, because $\mathrm{C}$ and $\varepsilon$-values affect the model in a different way and kernel parameters and $\mathrm{C}$ are dependent, the kernel function and all parameters cannot be chosen separately. There are different kernel functions are often found in the literature associated mapping process.

\subsubsection{Polynomial}

A polynomial mapping is a popular method for non-linear modeling. The second kernel is usually referable as it avoids problems with the hessian becoming Zero.

$$
\begin{aligned}
& K\left(x, x^{\prime}\right)=\left(x, x^{\prime}\right)^{d} \\
& K\left(x, x^{\prime}\right)=\left\{\left(x, x^{\prime}\right)+1\right\}^{d}
\end{aligned}
$$

\subsubsection{Gaussian Radial Basis Function}

Radial basis functions most commonly with a Gaussian form

$$
\mathrm{K}\left(\mathrm{x}, \mathrm{x}^{\prime}\right)=\exp \left(\frac{-\left\|\mathrm{x}-\mathrm{x}^{\prime}\right\|^{2}}{2 \sigma^{2}}\right)
$$

\subsubsection{Exponential Radial Basis Function}

A radial basis function produces a piecewise linear solution which can be attractive when discontinuities are acceptable

$$
\mathrm{K}\left(\mathrm{x}, \mathrm{x}^{\prime}\right)=\exp \left(\frac{-\left\|\mathrm{x}-\mathrm{x}^{\prime}\right\|}{2 \sigma^{2}}\right)
$$

\subsubsection{Sigmoidal Kernal}

The long established MLP, with a single hidden layer, also has a valid kernel representation

$\mathrm{K}\left(\mathrm{x}, \mathrm{x}^{\prime}\right)=\tanh \left(\rho\left(\mathrm{x}, \mathrm{x}^{\prime}\right)+l\right)$

For certain values of the scale, $\rho$, and offset 1 parameters. Here the Support Vectors correspond to the first layer and the Lagrange multipliers to the weights.

\section{NUMERICAL SIMULATION}

To evaluate the stability of the SVM PSS over a wide range of operating conditions, we consider typical example of a generator connected to infinite bus bar with the data

$\mathrm{K} 1=0.786, \mathrm{~K} 2=0.8644, \mathrm{~K} 3=0.3231, \mathrm{~T} 3=2.3567, \mathrm{~K} 5=-$ 0.1463 ,

$\mathrm{K} 6=0.4167$, Gex $(\mathrm{s})=\mathrm{KA}=200, \mathrm{KSTAB}=5, \mathrm{~T} 1=0.154 \mathrm{~s}$,

$\mathrm{T} 2=0.033 \mathrm{~s}, \mathrm{TW}=1.4 \mathrm{~s}, \mathrm{~K} 4=1.4189$.

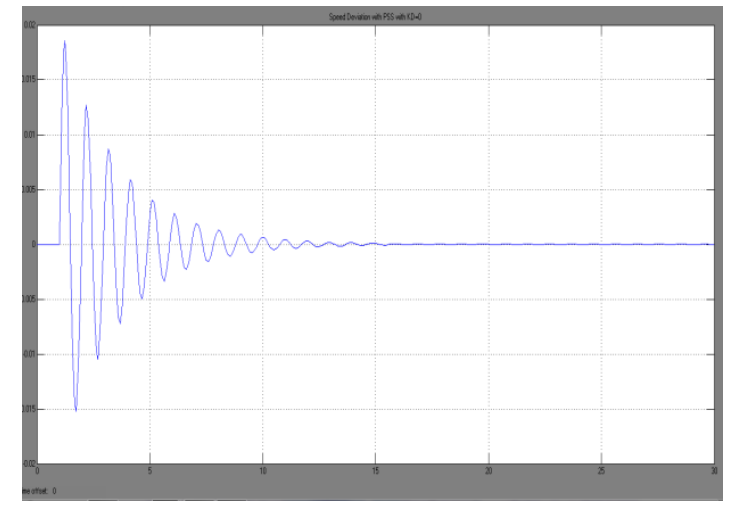

Fig 4:Speed Deviation versus time when $K_{D}=0$ with PSS

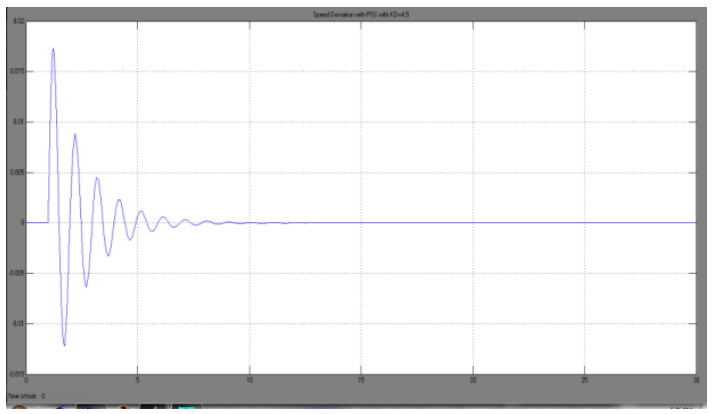

Fig 5: Speed Deviation versus time when $K_{D}=4.5$ with PSS

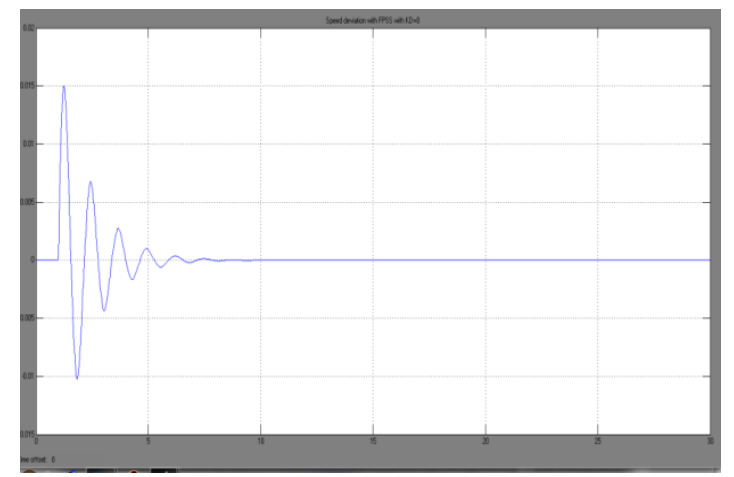

Fig. 6:Speed Deviation versus time when $K_{D}=0$ with Fuzzy PSS

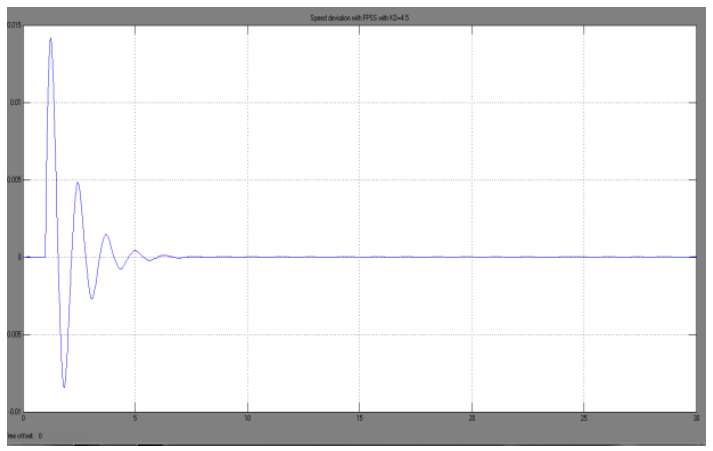

Fig 7:Speed Deviation versus time when $K_{D}=4.5$ with Fuzzy Based PSS 


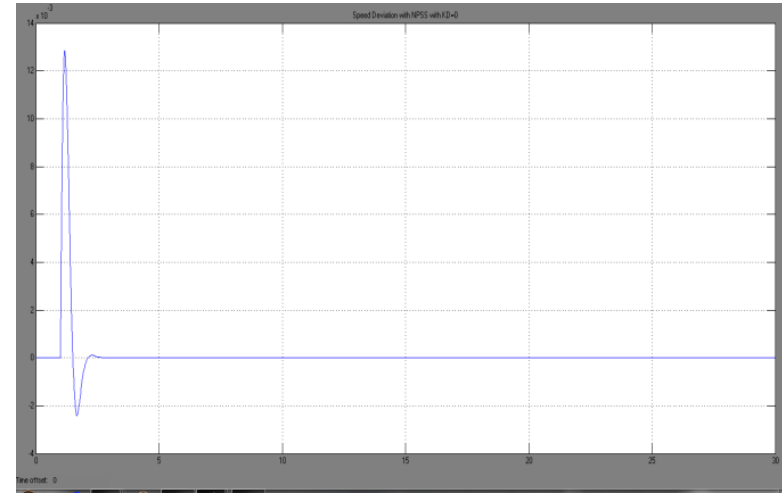

Fig 8:Speed Deviation versus time when $K_{D}=0$ with $S V M$ Based PSS

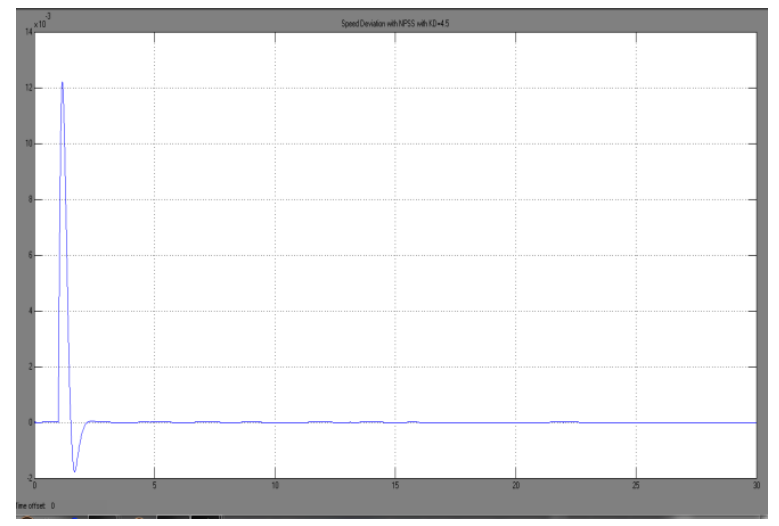

Fig. 9:Speed Deviation versus time when $K_{D}=4.5$ with SVM Based PSS

\section{CONCLUSION}

In this study, Support Vector Machine based Power System Stabilizer (SVMPSS) using sigmoidal kernel function and Fuzzy based PSS with triangular membership function is presented to adapt the PSS parameters to improve power system dynamic stability with same loading condition. SVMPSS with sigmodal kernel function gives good stability compared with Fuzzy based PSS and Conventional based PSS at different type of loading condition. The results show that the performance of the SVMPSS parameters yields the less settling time as compared with conventional and Fuzzy based PSS parameters.

\section{REFERENCES}

[1] Bhavans N.Shah, 2013."Comparative Study of Conventional And Fuzzy Based Power System Stabilizer" Computer Science and Network Technology
[2] Y.Butchi Raju, 2010. "Adaptive Power System Stabilizer Using Support Vector Machine" International Journal of Engineering Science and Technology

[3] D. K. Sambariya, R. Gupta, A. K. Sharma "Fuzzy Applications to Single Machine Power System Stabilizers" Journal of Theoretical And Applied Information Technology, ( 2005 - 2009 Jatit.

[4] E.A. Feilat,"Performance Comparison of Adaptive Estimation Techniques for Power System Small-Signal Stability Assessment", Proceedings of ICCCP 2009, Sultan Qaboos University, Feb 2009.

[5] G V Moodley, G D Jennings, R G Harley and M T Wishart, "Fuzzy Logic Power System Stabilizer in Multi Machine Stability Studies" IEEE Africon, Pp. 843-848, Sept. 1996

[6] H. A. Moussa, Y. Yu, "Optimal Power System Stabilization through Excitation and/or Governor Control", IEEE Transactions on Power Apparatus and Systems, Vol. PAS-91, pp1166-1174, 1972.

[7] K A El-Metwally, O P Malik, "Fuzzy Logic Based Power System Stabilizer", IEEE Proc- Gener.Transm. Distri., Vol. 142, No 3, May 1995.

[8] Jenica Ileana Corcau, Eleonor Stoenescu, Fuzzy Logic Controller as a Power System Stabilizer, International Journal Of Circuits, Systems And Signal Processing

[9] [9] C. Cortes and V. Vapnik. Support vector networks. Machine Learning, 20:273 - 297, 1995

[10] N. Aronszajn. Theory of reproducing kernels. Trans. Amer. Math. Soc., 686:337-404, 1950

[11] M. A. Aizerman, E. M. Braverman, and L. I. Rozono'er. Theoretical foundations of the potential function method in pattern recognition learning. Automation and Remote Control, 25:821-837, 1964.

[12] Burges C., "A tutorial on support vector machines for pattern recognition", In "Data Mining and Knowledge Discovery". Kluwer Academic Publishers, Boston, 1998, (Volume 2).

[13] C M Lim, "Application of A New Fuzzy Logic Control Scheme For Stability Enhancement- Simulation And Experimental Results", IEEE International Conference On Fuzzy Systems, Vol.3, Pp. 1339-1346, March 1995.

[14] A. Ghosh, G. Ledwich , O. P. Malik and G. S. Hope, "Power System Stabilizer Based on Adaptive Control Techniques", IEEE Transactions on Power Apparatus and Systems, Vol. PAS-103, No.8, August 1984. 